\title{
Preface: Celebrating vanadium science with leading bioinorganic contributions from the 9th International Vanadium Symposium
}

During the year of 2014, advances in vanadium science were celebrated at the International Vanadium Symposium in Italy in July and at the 248th American Chemical Society meeting in San Francisco in August. The vanadium symposium entitled "V9, 9th International Vanadium Symposium" was held for the first time in Italy, organized by the Chairwoman of the Vanadium Society Prof. Valeria Conte and Prof. Giulia Licini. The program consisted of approximately 100 oral and poster abstracts from vanadium scientists. This was the 9th in a series of symposia with a rich history summarized elsewhere $[1,2]$. These have been held since 1998 around the world with the two immediately preceding symposia being in Washington DC (Summer 2012) and in Toyama, Japan (Fall 2010). The 9th symposium was held at the "Centro Congressi San Gaetano" and the Archivio Antico of Padova University, near the Galileo Chair. The Italian vanadium chemists themselves have a long history of interest in vanadium beginning more than 50 years ago with Prof. Modena's contributions in organic synthesis using the metal as a catalyst [3-6]. The current volume of Journal of Inorganic Biochemistry compiles a number of contributions from both the vanadium conference and a symposium at the 248th American Chemic Society Meeting to honor Prof. Kenneth Kustin. We are particularly pleased to include in this volume the review by the Vanadis Awardee, Prof. Joao Costa Pessoa and the perspective on solution speciation by Prof. Kenneth Kustin who served the vanadium community for many years through his scientific endeavors and more recently as a chair of the Vanadis award committee. Finally, we are thrilled to include a review of the work by the late Lage Pettersson written by Prof. Rehder. During his lifetime, Prof. Pettersson resisted writing reviews and as such there is no previous compilation of his contributions to speciation of vanadium $(V)$. These three papers represent important contributions in vanadium science documenting how far the field has come and the quality of the investigation that continues in this field.

The 2014 Vanadis Award winner Professor Joao Costa Pessoa, from Lisbon, Portugal, has studied vanadium chemistry for more than three decades. He is one of the world's experts in EPR spectroscopy of vanadium and speciation of this metal. As the Director of "Centro de Química Estrutural (I.S.T.)" at Instituto Superior Técnico Universidade de Lisboa, Prof. Joao Pessoa has contributed to the vanadium science not only through his direct involvement but also by providing a supportive environment for young researchers entering the field. In accepting the award, he described his work over time beginning with solution studies characterizing the interactions of amino acids with the vanadyl cation. Prof. Pessoa also detailed more recent investigation into several controversial areas of vanadium science such as transferrin binding and transport, development of insulin-enhancing agents, development of vanadium-based catalysts, and applications to parasitic diseases.

In a review by Prof. Dieter Rehder, contributions to vanadium science by the late Lage Pettersson were summarized. For several decades, Prof. Pettersson was one of the main forces in the area of speciation; Prof. Tamas Kiss and he continued to assist in the development of speciation to a level of detail, which at present no other metal can rival. Prof. Pettersson began his interest in vanadium with inorganic systems. Working with several vanadium scientists including Oliver Howarth, Debbie C. Crans, Alan S. Tracey and Dieter Rehder, he became interested in biological systems and focused on the interactions of vanadium $(V)$ with buffers, amino acids and peptides, nucleosides and peroxide. His work was always of high quality and the field is grateful for his many contributions. The review of his research included in this volume will allow others to be familiarized with this work.

At the 248th ACS meeting, the scientific contributions by Prof. Kenneth Kustin were celebrated in a symposium honoring him. Important vanadium science has been advanced in part because of the involvement of Kenneth Kustin; this is particularly true in the areas of the dynamics of vanadium solution chemistry and science of vanadium-accumulating seasquirts. The review by Prof. Kustin describes in detail what is known regarding the dynamic processes of simple vanadium ions in solution. This contribution is a "must" read for anyone concerned with which species can be observed in solution and how they react.

Other contributions to this special issue are divided into five categories. The fundamental chemistry of vanadium in solution is the first category and this remains an important area in vanadium science since identifying complex formation and their reactivity is critical to understanding the complete system. The second category focuses on applications of vanadium compounds as anti-diabetic and anti-parasitic agents. These contributions include reviews as well as original studies probing mode of action and new vanadium compounds. The third category can be described broadly as metals in biology. These contributions cover mechanisms of toxicity, mode of action of anti-cancer agents and interactions with DNA and phosphatases. In addition, this section focuses on the molecular action at the vanadium center both in an enzyme (phosphatase) and in a functional mimic (nuclease activity). Contributions in the fourth category describe chemical conversions, some of which could be catalyzed by either vanadium compounds or another metal-related system. An example of novel materials based on two new polyoxometalates are included in this section. Finally, one contribution in the area of vanadium speciation and analysis is presented, one concerning speciation using XANES spectroscopy. Together, these 
articles provide a snapshot of the science that was presented at the International Vanadium Symposium in Padova in the summer of 2014.

In the section on fundamental chemistry, Prof. Anastasios Keramidas and coworkers tackle the problem of correlating properties of vanadium complexes with substituents. Specifically the team describes a simple donor atom additivity relationship, which they found can be used to calculate donor atom electrochemical potential. The electrochemical contributions of the square pyramidal vanadyl complexes were explored by the authors with the identification of specific relevant functionalities. These key functionalities are the OAc (acetylacetonate-enolic oxygen), OPh (phenolic oxygen), SPh (mercaptophenol sulfur), NAm (deprotonate amide nitrogen), NIm (imine nitrogen), Npy (pyridine nitrogen)

Pierluca Galloni and coworkers have extensively investigated vanadyl N,N'-bis(salicylidene)-o-phenylenediamine (salophen) complexes using cyclic voltammetry and UV-visible spectroscopy. The studies showed that substituents influence the metal electronic character. Because controverises associated with electron counting of vanadium compounds and the irreversibility of many vanadium compounds in water, the potential to use the cyclic voltammogram for design purposes is particularly noteworthy. A proposal is presented on how to design salophen ligands for vanadyl complexes with specific properties.

Prof. A. R. Rezvani and coworkers described the chemistry of vanadium-chelidamic acid and thiourea. These complexes build on the rich chemistry of vanadium dipicolinate complexes characterized when these compounds were investigated as insulin enhancing agents [7]. Some of the compounds were characterized and tested against both Gram-positive and Gram-negative bacterial strains with particular success. In the manuscript by Profs. Krivosudsky and Schwendt, the stereospecificity of vanadium complex formation was investigated. Specifically, the chiral anion $\left[\mathrm{VO}_{2}(\mathrm{~N}\right.$-salicylidene-isoleucinato $\left.)\right]$ with three chiral centers allowed for distinguishing between diastereomers with different crystallization times. It is important to note that the disparities in crystallization times corresponded to differences in solubility.

Vanadium compounds are known to exert insulin-enhancing effects and this is the topic of a number of contributions. Studies probing how these vanadium compounds interact with the blood proteins continue to be important because systemic distribution through the circulation is how these compounds reach different organs. The Garribba group report on the biotransformation of $\mathrm{VO}^{2+}$ complexes with ceftriaxone in red blood cells. How the 1:2 complex interacts with apo-transferrin was investigated using spectroscopic characterization and the ligands were found to dissociate when the vanadium binds to the protein. This mode of action is consistent with other reports [8,9], and the spectroscopic characterization of the species that forms allows for a comparison. Indeed, the studies with red blood cells show that this complex facilitates transport across the membrane.

The effects of a metforminium decavanadate salt on elevated lipid levels in Wistar rats was reported by the Gonzalez-Vergara group. Metformin is an anti-diabetic drug used in the clinic but, when combined in a salt with decavanadate, was found to induce the effects at much lower concentrations. This contribution described the anti-diabetic studies of this material. Seiichi Matsugo, Kan Kanamori, and coworkers review the historical background of vanadium chemistry and the physiological role of peroxidovanadium complexes and their interactions with insulin signal transduction pathways. Prof. A. Salifoglou and coworkers describe new anti-diabetic vanadodrugs based on the Schiff-base ligands. The compounds are soluble binary $\mathrm{V}(\mathrm{V})$ compounds that are cytotoxic based on their adipogenic activity in $3 \mathrm{~T} 3$-L1 fibroblasts toward mature adipocytes. The authors work to identify molecular targets and potential pathways involving the $\mathrm{V}(\mathrm{V})$-induced insulin-like activity.

Prof. Deborah Gambino, in collaboration with Prof. Joao Pessoa, reports the preparation of the family of heteroleptic oxidovanadium(IV) compounds with salicylaldehyde semicarbazones and polypyridyl ligands. The characterization of a range of new compounds, their properties, and their effects in biological systems are provided. The complexes are more active in vitro against Trypanosoma cruzi, the parasite responsible for Chagas disease, than the reference drug Nifurtimox. Several of the complexes are more active than previously reported for complexes of other NN co-ligands [10]. Whereas all the complexes interact well with DNA with binding modes and strength tuned in by the NN and semicarbazone co-ligands, molecular docking suggests the observed anti-T. cruzi activity cannot be explained by citing DNA intercalation as the sole mechanism of action.

The third category of contributions begins with a mechanistic pathway underlying inflammatory injury following exposures to vanadiumcontaining compounds. Andrew Ghio, Mitchell Cohen and coworkers hypothesize that the in vitro biological effect of vanadium results from an impact on iron homeostasis. Human bronchial epithelial cells exposed to vanadyl sulfate $\left(\mathrm{VOSO}_{4}\right)$ showed a time- and dosedependent increase in vanadium relative to the control phosphatebuffered-saline media. The human bronchial epithelial cells exposed to $\mathrm{VOSO}_{4}$ demonstrated significantly increased intracellular iron import following incubation with ferric ammonium citrate supporting an interaction between the two metals. It is postulated that an initiating event in biological effect after exposure to vanadyl sulfate is a loss of requisite cell iron.

Isabel Correia and coworkers synthesized, characterized, and tested copper(II) and vanadium(IV) Schiff base bipy and phen complexes against a range of cancer cells. The effects of the compounds are ligand- and metal-dependent. Some of the copper complexes show higher cytotoxic activity than both the corresponding vanadium complexes and cisplatin. Prof. Annette Rompel, Prof. Bernhard Keppler and coworkers reported studies on a series of binuclear vanadium complexes with group 12 metals. The synthesis of the vanadium - group 12 metal was described. The dinuclear complexes with $\mathrm{Zn}(\mathrm{II}), \mathrm{Hg}$ (II) and V(IV) were characterized by X-ray structural analysis. These complexes induce inhibition of protein tyrosine phosphatase 1B. Furthermore, the cytotoxicity of the complexes was determined in vitro using three cancer cell lines. Cytotoxic potency in the multidrug-resistant non-small cell lung cancer cell line A549 was at least as high as in the broadly chemosensitive ovarian teratocarcinoma cell line $\mathrm{CH} 1$ (PA-1).

Profs. Santiago Alvarez, Debbie C. Crans and coworkers describe the application of continuous shape analysis to investigate the structural space of the five-coordinate vanadium-phosphatase complexes that have been reported in the protein database [11]. The vanadium geometries are all found to be distorted trigonal bipyramidal (TBPY-5). No evidence for square pyramidal geometries was observed in any vanadiumprotein complex. Details associated with mechanistic transformations between the tetrahedral geometry and the five-coordinate high energy TBPY-5 geometry was discussed focusing on the protein tyrosine phosphatase 1B (PTP1B) enzyme. The umbrella distortions of the trigonal bipyramide are identified as being the most relevant types of transition state structures for the phosphoryl group transfer reactions for phosphatases and this may be related to the possibility that vanadium is an inhibitor for enzymes that support both dissociative exploded and associative five-coordinate transition states.

Prof. Isabel Cavaco and coworkers reported the effect of phosphate on the nuclease activity of oxidovanadium(IV) acetylacetonate and its derivatives. The ability to cleave DNA was studied by agarose gel electrophoresis, UV-vis spectroscopy, cyclic and square wave voltammetry, and ${ }^{51} \mathrm{~V}$ NMR spectroscopy. The mechanism is shown to be oxidative and associated with the formation of reactive oxygen species and faster than hydrolytic cleavage of the phosphodiester bond. A mixed-ligand complex containing phosphate, vanadium and acac was found to show a quasi-reversible voltammetric behavior and the implications of such an intermediate is discussed.

Turning to the fourth category of topics and the applications of vanadium compounds in synthesis and catalysis [12,13], papers describe contributions ranging from chlorination and iodination reactions to other different oxidation reactions and completing this section by one example of vanadium-containing oxometalate synthesis. Chlorination 
of ketones with dioxygen, in the presence of a vanadium catalyst and $\mathrm{Bu}_{4} \mathrm{NI}$ and $\mathrm{AlCl}_{3}$ was described by Moriuchi and coworkers. These conditions could also be used with alkenes to form vicinal chlorides. Finally the reaction of $\mathrm{Bu}_{4} \mathrm{NI}$ and $\mathrm{AlI}_{3}$ in the presence of a vanadium catalyst under atmospheric oxygen resulted in iodination of a substrate ketone. This reaction is particularly interesting since iodination is a very difficult process to accomplish cleanly but the vanadium catalyst might serve as a scaffold for these reactions [13]. The recyclable, stable, polymersupported (PS) oxidovanadium(IV) complex PS-[VIVO(sal-dahp)] is prepared by crosslinking polystyrene with $5 \%$ divinylbenzene and $\mathrm{H}_{3}$ sal-dahp. Prof. Mannar Maurya collaborating with Isabel Correia and coworkers reports that this material can be used for the peroxidase-like oxidation of pyrogallol.

Prof. Winfried Plass and coworkers describe a new vanadium(V) complex with a Schiff-base ligand containing a flexible amino side chain which can be protonated. The ligand was derived from salicylaldehyde and 6-aminohexanoic acid hydrazide hydrochloride and forms a vanadium complex by reacting with ammonium metavanadate. The complex catalyzes the oxidation of methylphenylsulfane to the corresponding sulfoxide in a methanol/dichloromethane mixture using hydrogen peroxide as oxidant. Prof. Jens Hartung and coworkers show that tert-butyl hydroperoxide stereoselectively oxidizes substituted 4-pentenols, when activated by (ethyl)[cis-(piperidine-2,6-diyl)dimethyl] vanadates. The reaction affords (tetrahydrofuran-2-yl)methanols in high yields and with moderate stereoselectivity. The 4-pentenols, differing by substitution at tetragonal and trigonal stereocenters, form products obtained from oxidative cyclization. A reaction model leading to stereoselectivity is provided involving the secondary coordination sphere.

Prof. Yoshihito Hayashi reports on the synthesis and characterization of fluoride-substituted polyoxovanadates increasing the versatility of the polyoxovanadates previously reported [14]. The presence of fluoride anion during the addition of acids exhibits strong intervention in the polyoxovanadate equilibria and two new polyoxovanadate species were identified by ${ }^{51} \mathrm{~V}$ NMR spectroscopy, $\left\{\mathrm{Et}_{4} \mathrm{~N}\right\}_{4}\left[\mathrm{~V}_{7} \mathrm{O}_{19} \mathrm{~F}\right]$ and $\left\{\mathrm{Et}_{4} \mathrm{~N}\right\}_{4}\left[\mathrm{HV}_{11} \mathrm{O}_{29} \mathrm{~F}_{2}\right]$. The polyanion $\left[\mathrm{V}_{7} \mathrm{O}_{19} \mathrm{~F}\right]^{4-}$ is the fluorideincorporated all $\mathrm{V}(\mathrm{V})$ state polyoxovanadate which has two different coordination environments of tetrahedral and square pyramidal vanadium units within the one anionic structural integrity. The structural gap, between tetrahedral-unit-based metavanadate and octahedralunit based decavanadate structure, may be linked by this hybrid complex. How effective these oxometalates may be as catalysts remains to be seen.

The final contribution in this special issue of Journal of Inorganic Biochemistry concerns speciation. Profs. Lay and Levina describe recent studies probing the effect of speciation on formulation of vanadium compounds using XANES spectroscopy. Speciation chemistry is here applied to the problem of drug formulation and this is a very interesting application. Indeed, such studies could be critical for applications of these compounds as a drug in the future. The use of carboxymethyl cellulose, xanthan gum or alginic acid in the formulations effect what species exist in the matrice and likely how the compound will act.

In summary, the 9th International vanadium symposium covered a wide range of topics in vanadium science worldwide. The year 2014 will remain a special year with all the events that took place for vanadium scientists, and the current volume describe some of that chemistry. The 10th International vanadium symposium will be in Taipei, Taiwan in 2016. We look forward to seeing the next level of research in vanadium science that the future will bring.

\section{References}

[1] C.C. McLauchlan, D.C. Crans, Daltons Trans. 42 (2013) 11744-11748.

[2] H. Michibata, K. Kanamori, T. Hirao, Coord. Chem. Rev. 255 (2011) 2149.

[3] R. Curci, F. DiFuria, R. Testi, G. Modena, J. Chem. Soc. Perkin Trans. 2 (1974) 752-757.

[4] S. Cenci, F. DiFuria, G. Modena, R. Curci, J.O. Edwards, J. Chem. Soc. Perkin Trans. 2 (1978) 979-1086.

[5] V Conte, F. DiFuria, G. Modena, J. Org Chem 53 (1988) 1665-1669.

[6] V. Conte, F. DiFuria, G. Licini, Appl. Catal. 157 (1997) 335-361.

[7] G.R. Willsky, L.-H. Chi, M.E. Godzalla III, P.J. Kostyniak, J.J. Smee, A.M. Trujillo, J.A. Alfano, W. Ding, Z. Hu, D.C. Crans, Coord. Chem. Rev. 19-20 (2011) 2258-2269.

[8] T. Jakusch, J. Costa Pessoa, T. Kiss, Coord. Chem. Rev. 255 (2011) 2218-2226

[9] D. Sanna, L. Biro, P. Buglyo, G. Micera, E. Garribba, Metabollomics 4 (2012) 33-36.

[10] M. Fernández, J. Varela, I. Correia, E. Birriel, J. Castiglioni, V. Moreno, J. Costa Pessoa, H. Cerecetto, M. González, D. Gambino, Daltons Trans. 42 (2013) 11900-11911.

[11] C. C. McLauchlan, B. J. Peters, G. R. Willsky and D. C. Crans Coord. Chem. Rev. doi:10. 1016/j.ccr.2014.12.012.

[12] G. Licini, V. Conte, A. Coletti, M. Mba, C. Zonta, Coord. Chem. Rev. 255 (2011) 2345-2357.

[13] T. Moriuchi, T. Hirao, Chem. Soc. Rev. 33 (2004) 294-301.

[14] Y. Hayashi, Coord. Chem. Rev. 255 (2011) 2270-2280.

Debbie C. Crans Dept. Chemistry and Cell and Molecular Biology, Colorado State University, Fort Collins, Colorado 80523 Corresponding Author. E-mail address: Debbie.Crans@ColoState.Edu.

Andrew Ghio National Health and Environmental Effects Research Laboratory, Chapel Hill, NC 27599

Valeria Conte Department of Chemical Sciences and Technologies, University of Roma Tor Vergata, via Ricerca Scientifica, snc, 00133 Roma, Italy 